\title{
Intoxicação experimental aguda por Senecio brasiliensis em ovinos e indução de resistência à intoxicação ${ }^{1}$
}

\author{
Fabiane B. Grecco ${ }^{2}$, Pablo Estima-Silva ${ }^{3}$, Clairton Marcolongo-Pereira ${ }^{4}$, Mauro P. \\ Soares ${ }^{5}$, Margarida B. Raffi ${ }^{2}$, Eliza S.V. Sallis ${ }^{2}$ e Ana Lucia Schild ${ }^{5 *}$
}

\begin{abstract}
Grecco F.B., Estima-Silva P., Marcolongo-Pereira C., Soares M.P., Raffi M.B. \& Schild A.L. 2012. [Acute experimental poisoning by Senecio brasiliensis in sheep and induced resistance against the poisoning.] Intoxicação experimental aguda por Senecio brasiliensis em ovinos e indução de resistência à intoxicação. Pesquisa Veterinária Brasileira 32(9):912-916. Laboratório Regional de Diagnóstico, Faculdade de Veterinária, Universidade Federal de Pelotas, Campus Universitário s/n, Pelotas, RS 96010-900, Brazil. E-mail: alschild@terra.com.br

Sheep are more resistant to poisoning by Senecio spp. than cattle and horses. To determine whether this resistance could be induced by ingestion of small and repeated doses of the plant and to know how long the resistance could last, three experiments with fresh green leaves and stalks of Senecio brasiliensis were conducted. In Experiment 1, to determine the minimum dose for acute poisoning, single doses of $60,80,90,100$ and $100 \mathrm{~g} / \mathrm{kg}$ body weight (bw) were administered to five 1 and a half-year-old male Corriedale sheep. The sheep fed $60 \mathrm{~g} / \mathrm{kg}$ bw and $80 \mathrm{~g} / \mathrm{kg}$ bw of $S$. brasiliensis did not get sick; however, the sheep that received $80 \mathrm{~g} / \mathrm{kg}$ bw, developed mild fibrosis and megalocytosis in liver biopsies performed 90,120 and 150 days after the end of plant administration. The sheep that received 90 and $100 \mathrm{~g} / \mathrm{kg}$ bw showed clinical signs of anorexia, prostration, paddling movements and abdominal pain, and died 12-48 hours after the onset of clinical signs. At their necropsy, ascites, disseminated petechiae and accentuated lobular pattern of the liver were found, and histologicaly hemorrhagic centrilobular necrosis was observed. In Experiment 2 , the dose of $100 \mathrm{~g} / \mathrm{kg}$ bw fractionated into 2,5 and 10 daily doses was administered to three sheep respectively. The fractioned dosage did not cause clinical signs of poisoning in any of the sheep, but periportal fibrosis and moderate megalocytosis were observed in liver biopsy performed at day 60 after the end of the plant administration. No histological lesions were observed in hepatic biopsies of sheep that received 10 daily doses of $10 \mathrm{~g} / \mathrm{kg}$ bw. In Experiment 3, to determine whether the sheep become resistant to the acute form of poisoning, four sheep ingested daily doses of $15 \mathrm{~g} / \mathrm{kg}$ bw of $S$. brasiliensis during 30 days and daily doses of $30 \mathrm{~g} / \mathrm{kg}$ bw for 10 days. On the day following the last dose of $30 \mathrm{~g} / \mathrm{kg}$ bw, two sheep were challenged with $100 \mathrm{~g} / \mathrm{kg}$ bw of the plant in a single dose. No clinical signs were observed in both sheep and no histological lesions could be found in the liver biopsies obtained 15 and 30 days after administration of the challenge dose. The third and fourth sheep received the challenge dose $(100 \mathrm{~g} / \mathrm{kg}$ bw $) 15$ and 45 days after the end of plant administration. These animals showed anorexia, abdominal pain, and died about 12 hours after the onset of clinical signs. Gross and microscopic lesions were similar to those observed in sheep from Experiment 1. The results of this study demonstrate that sheep are susceptible to acute S. brasiliensis poisoning at doses of 90 and $100 \mathrm{~g} / \mathrm{kg}$ bw, but they beco-
\end{abstract}

\footnotetext{
${ }^{1}$ Recebido em 23 de fevereiro de 2012.

Aceito para publicação em 8 de junho de 2012.

${ }^{2}$ Docentes do Departamento de Patologia Animal, Faculdade de Veterinária, Universidade Federal de Pelotas (UFPel), Campus Universitário s/n, Pelotas, RS 96010-900, Brasil.

${ }^{3}$ Graduando de Medicina Veterinária, Faculdade de Veterinária, UFPel,
}

\footnotetext{
Pelotas, RS. Bolsista de Iniciação Científica, Conselho Nacional de Desenvolvimento Científico e Tecnológico (CNPq).

${ }^{4}$ Pós-Graduando em Veterinária, Faculdade de Veterinária, UFPel, Pelotas, RS.

${ }^{5}$ Médicos Veterinários do Laboratório Regional de Diagnóstico, Faculdade de Veterinária, UFPel, Pelotas, RS. *Autor para correspondência: alschild@terra.com.br
} 
me resistant after the continuous ingestion of small daily doses. This resistance has been lost 15 days after the end of the plant ingestion. Natural acute poisoning by $S$. brasiliensis probably does not occur in sheep, because they would have to eat large amounts of the plant in a short period of time.

INDEX TERMS: Poisonous plants, Senecio brasiliensis, hepatotoxicosis, pyrrolizidine alkaloids, plant poisoning, sheep.

RESUMO.- Ovinos são mais resistentes à intoxicação por Senecio spp. que bovinos e equinos. Para determinar se essa resistência é induzida pela ingestão de pequenas e repetidas doses da planta e se essa resistência é duradoura, foram realizados três experimentos com folhas e talos verdes de Senecio brasilienses. Para determinar a dose mínima que causa intoxicação aguda (experimento 1 ), foram administradas doses únicas de $60,80,90,100$ e $100 \mathrm{~g} / \mathrm{kg}$ de peso corporal (pc) a cinco ovinos, respectivamente. Os animais que receberam 60 e $80 \mathrm{~g} / \mathrm{kg}$ de pc de $S$. brasiliensis não adoeceram, porém o ovino que recebeu $80 \mathrm{~g} / \mathrm{kg}$ de pc apresentava fibrose e megalocitose discretas nas biópsias realizadas aos 90, 120 e 150 dias do término da administração da planta. Os ovinos que receberam 90 e $100 \mathrm{~g} / \mathrm{kg}$ de pc apresentaram anorexia, prostração, movimentos de pedalagem, dor abdominal e morte 12-48 horas após o aparecimento dos sinais clínicos. Na necropsia havia ascite, petéquias disseminadas e acentuação do padrão lobular hepático. Histologicamente havia necrose hemorrágica centro-lobular. No Experimento 2 a dose capaz de causar a intoxicação aguda foi fracionada e administrada em duas, cinco e 10 doses diárias para 3 ovinos, respectivamente. A dose tóxica fracionada não provocou sinais clínicos de intoxicação em nenhum dos ovinos, porém havia fibrose periportal e megalocitose moderadas nas biopsias realizadas aos 60 dias do término da administração da planta, as quais não evoluíram. 0 ovino que recebeu a dose fracionada em 10 administrações não apresentou lesões histológicas nas biópsias. Para determinar se os ovinos tornam-se resistentes à forma aguda da intoxicação (experimento 3), foram administradas doses diárias de $15 \mathrm{~g} /$ $\mathrm{kg}$ de pc por 30 dias e $30 \mathrm{~g} / \mathrm{kg}$ de pc por 10 dias a quatro ovinos. No dia seguinte à última administração dois ovinos receberam a dose única de $100 \mathrm{~g} / \mathrm{kg}$ de pc de $S$. brasiliensis, mas não adoeceram nem apresentaram lesões em biópsias realizadas 15 e 30 dias após o desafio. Entretanto, dois ovinos que receberam essa dose, 15 e 45 dias após a última administração da planta, respectivamente, apresentaram anorexia, dor abdominal, movimentos de pedalagem e morreram 12 horas após o início dos sinais clínicos. As lesões macro e microscópicas eram semelhantes às observadas nos ovinos do Experimento 1. Os resultados do presente trabalho demonstraram que, experimentalmente, ovinos intoxicam-se de forma aguda com doses únicas de $90-100 \mathrm{~g} / \mathrm{kg}$ de pc, mas são capazes de suportar essas doses após a ingestão contínua de pequenas quantidades da planta, no entanto, essa suposta resistência é perdida se os ovinos deixam de ingerir a mesma. Pode-se sugerir também que a intoxicação aguda natural, provavelmente, não ocorre devido à improbabilidade de ingestão, por um ovino, da quantidade necessária de planta em um período curto de tempo.
TERMOS DE INDEXAÇÃO: Plantas tóxicas, Senecio brasiliensis, hepatotoxicose, alcalóides pirrolizidínicos, intoxicação por plantas, ovinos.

\section{INTRODUÇÃO}

Tem sido estimado que a morte de animais no Rio Grande do Sul em consequência das intoxicações por plantas representam uma perda anual de aproximadamente 21 milhões de dólares (Riet-Correa \& Medeiros 2000, Rissi et al 2007). Essas perdas podem ser bem maiores se incluirmos nesse cálculo os gastos associados com o controle das plantas tóxicas. Nesse contexto, Senecio spp. é a planta tóxica mais importante do estado e é responsável por $50 \%$ de todas as mortes de bovinos em decorrência de intoxicações. Dentre as espécies do gênero Senecio, S. brasiliensis (Maria-mole) é uma das mais difundidas no estado do Rio Grande do Sul (Karam et al. 2002). S. oxyphyllus, S. heterotrichius, S. selloi, S. tweediei (Méndez \& Riet-Correa 1993) e S. madagascariensis (Cruz et al. 2010) são, também, associadas com casos de intoxicação em bovinos.

Alternativas de controle e profilaxia das intoxicações por Senecio spp. são, constantemente procuradas por criadores e profissionais da área. 0 pastoreio por ovinos e caprinos em áreas invadidas por plantas do gênero Senecio é uma das práticas mais utilizadas (Dollhite 1972). Os ovinos são tidos como mais resistentes à intoxicação por alcalóides pirrolizidínicos (APs) presentes nas espécies de Senecio, de modo que podem consumir e controlar a planta sem adoecer na maioria das vezes (Méndez \& Riet-orrea 1993).

Os APs em ovinos podem causar intoxicação aguda com necrose centrolobular e intoxicação crônica com fibrose hepática, fotossensibilização, encefalopatia hepática e edemas, ou intoxicação crônica por cobre, hemólise, icterícia e hemoglobinúria (Seaman 1987, Ilha et al 2001, Kellerman et al 2005, Nobre et al 2005, Grecco et al. 2011). No Brasil, surtos de intoxicação por Senecio spp em ovinos são raros, apesar de frequentes em outras partes do mundo (Bull et al.1968, Forsyth 1979, Kellerman et al. 2005). A ocorrência de surtos de seneciose em ovinos no Rio Grande do Sul (Ilha et al. 2001, Grecco et al. 2011) indica a necessidade de estudos adicionais, especialmente os associados com a resistência dos ovinos à intoxicação para utilizar com segurança esta espécie animal no controle de Senecio spp.

0 presente trabalho teve como objetivos determinar a dose de Senecio brasiliensis capaz de produzir intoxicação aguda em ovinos e comprovar que doses pequenas e repetidas da planta são capazes de induzir a resistência dos animais à intoxicação.

\section{MATERIAL E MÉTODOS}

Para a realização dos experimentos foram utilizados folhas e talos verdes de Senecio brasiliensis colhidos no município de Pelotas, Rio Grande do Sul, imediatamente antes da administração. 
'Determinação da dose tóxica para causar intoxicação aguda (Exp.1)

Foram administradas doses de $60 \mathrm{~g}, 80 \mathrm{~g}$ e $100 \mathrm{~g} / \mathrm{kg}$ de peso corporal (pc) de folhas frescas de $S$. brasiliensis em dose única para 3 ovinos Corriedale, machos de 1,5 anos de idade (Ovinos 1, 2 e 3) até que se reproduzisse a forma aguda da intoxicação. Posteriormente dois outros animais da mesma raça e idade (Ovinos 4 e 5) receberam $90 \mathrm{~g}$ e $100 \mathrm{~g} / \mathrm{kg}$ de pc da planta, também, em dose única. Nos ovinos que não apresentaram sinais de intoxicação foram realizadas biópsias hepáticas aos 90,120 e 150 dias do término da administração da planta. No Quadro 1 é apresentado o protocolo utilizado no experimento.

Fracionamento da dose tóxica que induz intoxicação aguda (Exp.2)

A partir da determinação da dose de planta que causa intoxicação aguda, três ovinos Corriedale machos de 1,5 anos de idade (Ovinos 6, 7 e 8), receberam a dose de planta suficiente para causar a intoxicação aguda dividida em duas, cinco e 10 doses administradas diariamente durante dois, cinco e 10 dias, respectivamente. Os dados deste experimento encontram-se no Quadro 2. Foram realizadas biópsias hepáticas dos Ovinos aos 30, 60, 90 e 150 dias após administração da última dose de planta.

\section{Indução de resistência em ovinos à intoxicação por Senecio brasiliensis (Exp.3)}

Quatro animais (Ovinos 9, 10, 11 e 12), três machos e uma fêmea de oito meses de idade, receberam $15 \mathrm{~g} / \mathrm{kg}$ de $\mathrm{pc}$ de folhas e talos de $S$. brasiliensis diariamente por 30 dias e $30 \mathrm{~g} / \mathrm{kg}$ de pc por 10 dias. No dia seguinte a última administração de $30 \mathrm{~g} / \mathrm{kg}$ de pc, os Ovinos 9 e 10 foram submetidos a desafio com a dose tóxica causadora da forma aguda da intoxicação. Os ovinos foram observados diariamente para a detecção de qualquer sinal clínico de intoxicação. Biópsias hepáticas foram realizadas aos 15 e 30 dias do início experimento e 15 dias após o desafio com a dose tóxica. Os Ovinos 11 e 12 foram desafiados aos 15 e 45 dias após a ingestão da última dose de $30 \mathrm{~g} / \mathrm{kg}$ de pc, respectivamente. No Quadro 3 é apresentado um resumo do protocolo utilizado.

Quadro 1. Peso dos animais, dose administrada, número de doses, dose total e desfecho do experimento de reprodução da intoxicação aguda por Senecio brasiliensis em ovinos

\begin{tabular}{|c|c|c|c|c|c|}
\hline Ovinos & $\begin{array}{l}\text { Peso } \\
\text { (kg) }\end{array}$ & $\begin{array}{c}\text { Dose } \\
\text { (g/kg) }\end{array}$ & №de doses & $\begin{array}{l}\text { Dose total } \\
(\mathrm{kg})\end{array}$ & Desfecho \\
\hline 1 & 23 & 60 & 1 & 1,380 & Eutanásia $^{\mathrm{a}}$ \\
\hline 2 & 25 & 80 & 1 & 2,000 & Eutanásia $^{\mathrm{a}}$ \\
\hline 3 & 24 & 100 & 1 & 2,400 & Morte em 12 horas \\
\hline 4 & 24 & 100 & 1 & 2,400 & Morte em 24 horas \\
\hline 5 & 23 & 90 & 1 & 2,070 & Morte em 4 horas \\
\hline
\end{tabular}

a Eutanásia aos 210 dias do término da administração de Senecio brasilien-

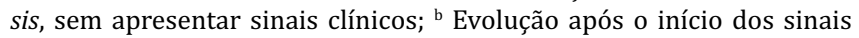
clínicos.

Quadro 2. Peso dos animais, dose, número de doses, dose total e desfecho do experimento com fracionamento da dose que induz intoxicação aguda por Senecio brasiliensis em ovinos

\begin{tabular}{cccccc}
\hline Ovinos & Peso $(\mathrm{kg})$ & Dose $(\mathrm{g} / \mathrm{kg})$ & № de doses & Dose total $(\mathrm{kg})$ & Desfecho \\
\hline 6 & 20 & 50 & 2 & 2,000 & Eutanásia $^{\mathrm{a}}$ \\
7 & 21,5 & 20 & 5 & 2,150 & Eutanásia $^{\mathrm{a}}$ \\
8 & 23 & 10 & 10 & 2,300 & Eutanásia $^{\mathrm{a}}$
\end{tabular}

a Eutanásia aos 230 dias do término da administração de Senecio brasiliensis sem apresentar sinais clínicos.
Quadro 3. Peso dos animais, dose de planta administrada, número de doses, dose total e desfecho do experimento de indução de resistência de ovinos à intoxicação por Senecio brasiliensis

\begin{tabular}{|c|c|c|c|c|c|c|}
\hline Ovinos & $\begin{array}{c}\text { Peso } \\
\text { (kg) }\end{array}$ & $\begin{array}{c}\text { Dose } \\
\text { (g/kg) }\end{array}$ & $\begin{array}{l}\text { № de } \\
\text { doses }^{\mathrm{a}}\end{array}$ & $\begin{array}{l}\text { Dose } \\
\text { total } \\
(\mathrm{kg})\end{array}$ & $\begin{array}{c}\text { Período do } \\
\text { desafio após } \\
\text { última dose } \\
\text { de } 10 \mathrm{~g} / \mathrm{kg}\end{array}$ & $\begin{array}{c}\text { Desfecho } \\
\text { após o } \\
\text { desafio }\end{array}$ \\
\hline \multirow[t]{3}{*}{9} & \multirow[t]{3}{*}{21} & 15 & 30 & 9,450 & \multirow[t]{3}{*}{1 dia } & \multirow[t]{3}{*}{ Eutanásiab $^{\mathrm{b}}$} \\
\hline & & 30 & 10 & 4,410 & & \\
\hline & & 100 & 1 & 2,100 & & \\
\hline \multirow[t]{3}{*}{10} & \multirow[t]{3}{*}{24} & 15 & 30 & 10,800 & \multirow[t]{3}{*}{1 dia } & \multirow[t]{3}{*}{ Eutanásiab $^{\mathrm{b}}$} \\
\hline & & 30 & 10 & 7,200 & & \\
\hline & & 100 & 1 & 2,400 & & \\
\hline \multirow[t]{3}{*}{11} & \multirow[t]{3}{*}{23} & 15 & 30 & 10,350 & \multirow{3}{*}{15 dias } & \multirow{6}{*}{$\begin{array}{l}\text { Morte } 6 \text { horas } \\
\text { após o desafio } \\
\text { Morte } 6 \text { horas } \\
\text { após o desafio }\end{array}$} \\
\hline & & 30 & 10 & 6,900 & & \\
\hline & & 100 & 1 & 2,300 & & \\
\hline \multirow[t]{3}{*}{12} & \multirow[t]{3}{*}{22} & 15 & 30 & 9,900 & \multirow[t]{3}{*}{45 dias } & \\
\hline & & 30 & 10 & 6,600 & & \\
\hline & & 100 & 1 & 2,200 & & \\
\hline
\end{tabular}

${ }^{a}$ Os ovinos receberam Senecio brasiliensis em dias corridos a partir de 10 de agosto de 2011; ' ${ }^{\text {E}}$ Eutanásia aos 120 dias após o desafio, sem sinais clínicos.

Os ovinos que não morreram espontaneamente foram eutanasiados aos 210 dias do fim da administração da planta. Os animais foram necropsiados e fragmentos dos órgãos das cavidades abdominal e torácica e o sistema nervoso central, foram fixados em formalina a $10 \%$, processados rotineiramente para histopatologia e corados pela hematoxilina-eosina.

\section{RESULTADOS}

\section{Determinação da dose tóxica para causar intoxicação aguda (Exp.1)}

O Ovino 1 não mostrou sinais clínicos da intoxicação e não foram observadas lesões histológicas nas biópsias hepáticas realizadas 90 e 120 dias após o término da administração. 0 Ovino 2 não apresentou sinais clínicos da intoxicação, porém observou-se megalocitose e fibrose discretas na biópsia realizada 120 dias após o término da administração e que não progrediram na biópsia realizada aos 150 dias. 0 Ovino 1, eutanasiado aos 210 dias do término do experimento, não apresentava lesões macro ou microscópicas significativas. 0 Ovino 2 não apresentava lesões macroscópicas e histologicamente havia fibrose periportal moderada, megalocitose e nódulos regenerativos em fase de desenvolvimento. Os Ovinos 3, 4 e 5 apresentaram anorexia, decúbito lateral com movimentos de pedalagem e sinais de dor abdominal 22-24 horas após a administração da planta. 0 Ovino 4 morreu 24 horas após o início dos sinais clínicos. Os Ovinos 3 e 5 morreram entre 2 e 4 horas do início dos sinais clínicos, respectivamente. Na necropsia havia ascite acentuada, petéquias disseminadas pelo omento e mesentério (Fig.1), edema de abomaso, fígado friável e com acentuação do padrão lobular (Fig.2). Na histopatologia havia necrose hemorrágica centro-lobular acentuada. Nos demais órgãos não foram observadas lesões. 0 desfecho do experimento é apresentado no Quadro 1.

\section{Fracionamento da dose tóxica que induz intoxicação aguda (Exp.2)}

Os Ovinos 6 e 7 não apresentaram sinais clínicos de intoxicação durante o experimento. Histologicamente havia 
moderada proliferação de ductos biliares nas biópsias hepáticas realizadas 60 dias após a administração da planta. Esta lesão era mais acentuada e acompanhada de fibrose periportal e megalocitose moderadas nos dois ovinos nas biópsias realizadas 90 dias após a administração da planta. Nas biópsias realizadas 150 dias após as lesões não progrediram. Os Ovinos 6 e 7 foram eutanasiados aos 210 dias do final da administração da planta e não foram observadas lesões macroscópicas. Microscopicamente, o Ovino 6 apresentava moderada megalocitose de hepatócitos.

O Ovino 8 não apresentou lesões microscópicas da intoxicação na biópsia hepática realizada aos 30 dias do início do experimento. 0 desfecho deste experimento é apresentado no Quadro 2.

\section{Indução de resistência em ovinos à intoxicação por Se- necio brasiliensis (Exp.3)}

Os Ovinos 9 e 10 desafiados com $100 \mathrm{~g} / \mathrm{kg}$ de pc de planta verde, após a administração da planta não apresentaram sinais clínicos de intoxicação. As biópsias realizadas aos 15 e 30 dias após o desafio não apresentaram alterações microscópicas. Os animais foram eutanasiados aos 230 dias do início do experimento e não havia lesões macroscópicas

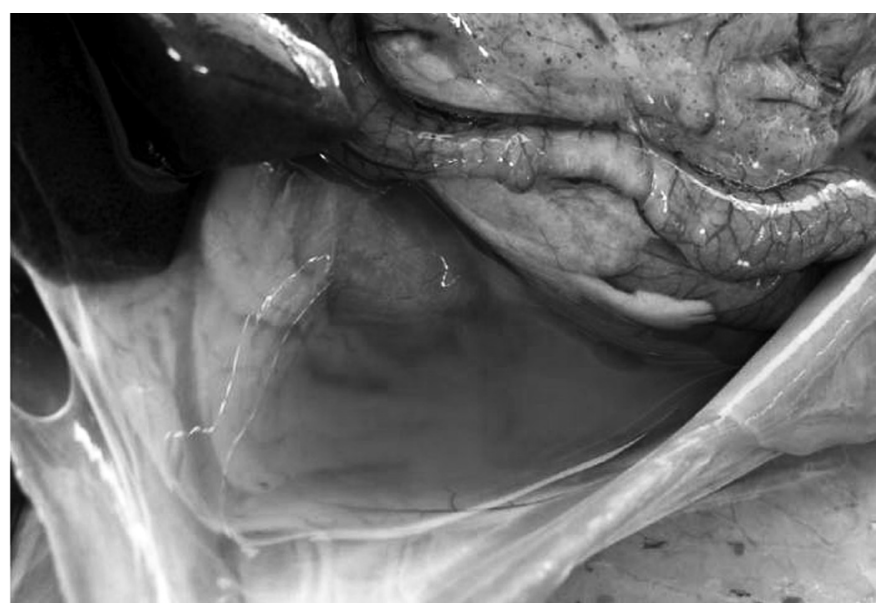

Fig.1. Ascite e petéquias no mesentério, na intoxicação experimental aguda por Senecio brasiliensis (Ovino 3). ou histológicas. Os Ovinos 11 e 12 apresentaram sinais clínicos de depressão 22-24 horas após a ingestão da planta e morreram 4-6 horas após o aparecimento dos sinais clínicos respectivamente. $\mathrm{Na}$ necropsia o fígado de ambos animais estava escuro e havia discreta acentuação do padrão lobular. Histologicamente havia necrose hemorrágica centrolobular. 0 desfecho do experimento é apresentado no Quadro 3.

\section{DISCUSSÃO}

Os resultados dos experimentos realizados demonstram que ovinos, apesar de mais resistentes à intoxicação por Senecio brasiliensis do que os bovinos e equinos, são suscetíveis à forma aguda da intoxicação ao ingerir dose única mínima de $90 \mathrm{~g} / \mathrm{kg}$ peso corporal (pc) de planta verde. Por outro lado, a intoxicação aguda espontânea em ovinos nas condições de pastoreio, provavelmente não ocorre uma vez que dificilmente um ovino chegará a ingerir espontaneamente $90 \mathrm{~g} / \mathrm{kg}$ de pc em um período curto de tempo. Desta forma, em lotações adequadas, a utilização dos ovinos no controle da população de Senecio spp. pode ser eficiente uma vez que os animais normalmente ingerem pequenas quantidades diárias da planta e graças a sua ampla capacidade de detoxificar os alcaloides pirrolizidínicos (APs) e por particularidades de sua flora ruminal, eliminam mais facilmente estes alcaloides não sofrendo danos hepáticos (Laningan 1970, Huan et al 1998).

Deve ser mencionado, porém, que a intoxicação crônica por Senecio spp. foi reproduzida experimentalmente em ovinos pela administração do equivalente a $2,5 \mathrm{~g} / \mathrm{kg}$ de pc de planta verde por 60 dias (Barros et al. 1989), o que sugere cautela no uso dessa espécie para o controle da planta. A intoxicação crônica espontânea pode ocorrer quando ovinos permanecem por períodos prolongados em lotações inadequadas em pastagens severamente invadidas (Ilha et al. 2001, Grecco et al. 2011).

Em um trabalho de reprodução experimental da intoxicação em ovinos por $S$. erraticus no Chile os autores mencionaram que muitos animais não apresentavam sinais clínicos e/ ou lesões macroscópicas, mas apresentavam lesões histológicas características e atribuíram este fato a resistência maior dessa espécie à intoxicação (Araya et al. 1983). No presente
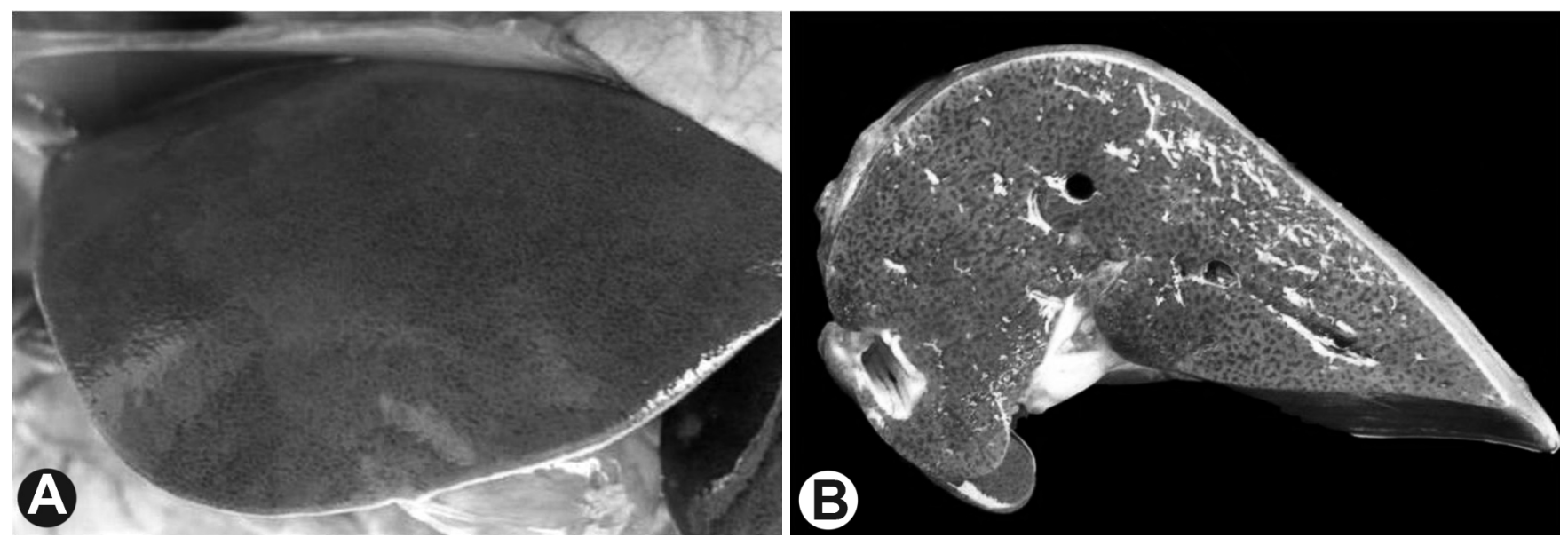

Fig.2. (A) Acentuação do padrão lobular hepático na superfície capsular e (B) de corte, na intoxicação experimental aguda por Senecio brasiliensis (Ovino 5). 
trabalho, o Ovino 2 (Experimento 1) e os Ovinos 6 e 7 (Experimento 2) apresentavam lesões histológicas discretas da intoxicação por APs, que progrediram até 120 dias após o início do experimento. Isto parece sugerir que em condições de campo os ovinos podem apresentar lesões histológicas discretas, porém não são observados sinais clínicos em virtude de que não há comprometimento de mais de $75 \%$ do fígado, condição indispensável para o desenvolvimento de insuficiência hepática. Outro fator que pode estar relacionado a não ocorrência da intoxicação com maior frequência é o tempo de vida produtiva curto da espécie ovina dentro das propriedades.

Os resultados do Experimento 3 (Ovinos 9 e 10) mostraram que ovinos são capazes de suportar a dose que induz intoxicação aguda se desafiados com essa dose imediatamente após a ingestão de doses diárias de $15 \mathrm{~g} / \mathrm{kg}$ de pc por 30 dias e $30 \mathrm{~g} / \mathrm{kg}$ por 10 dias. Por outro lado, os Ovinos 11 e 12 morreram ao serem desafiados 15 e 45 dias após ter cessado a ingestão fracionada de $S$. brasiliensis. Isso sugere que para que se mantenha essa suposta resistência é necessária a ingestão contínua da planta e a resistência é perdida rapidamente ao cessar a ingestão. Provavelmente, isto ocorre porque os hepatócitos durante a injúria provocada pelos APs estão sofrendo adaptações benéficas em seu metabolismo de reparação e detoxificação visando restaurar a homeostasia do funcionamento hepático, através do maior nível de enzimas microssomais adaptadas a essas substâncias (Cullen 2007). Por outro lado, se o estímulo é removido os hepatócitos retornam ao seu estado fisiológico anterior pelo decréscimo de proteínas específicas e há degradação lissosomal das enzimas adaptadas. Em consequência, no momento do desafio os ovinos não conseguem detoxificar a carga excessiva de APs ingeridos e se intoxicam.

Anjos et. al. (2009), em experimentos com sementes de Crotalaria retusa em ovinos demonstraram, também, que esta espécie adquire resistência ingerindo pequenas doses da planta, porém estes autores não testaram quanto tempo a resistência permanece após cessar a ingestão da mesma.

\section{CONCLUSÕES}

Os resultados obtidos indicam que a resistência dos ovinos à intoxicação por Senecio brasiliensis está associada à ingestão contínua de pequenas quantidades da planta.

No entanto, essa suposta resistência é perdida se os ovinos deixam de ingerir a mesma.

Há pouca possibilidade que a intoxicação aguda ocorra nos ovinos em condições naturais, pois dificilmente ingerem quantidades necessárias de planta em um período curto de tempo.

Agradecimentos.- Trabalho financiado pelo INCT/CNPq (Proc.573534/ 2008-0).

\section{REFERÊNCIAS}

Anjos B.L., Nobre V.M.T., Dantas A.F.M., Medeiros R.M.T., Oliveira Neto T.S., Molineux R.J. \& Riet-Correa F. 2009. Poisoning of sheep by seeds of Crotalaria retusa: Adquired resistance by continuous administration of low doses. Toxicon 55:28-32.

Araya O., Hernandez J.R., Espinoza A.E. \& Cubillos V. 1983. Serum changes and histologic liver lesions due to experimental ingestion of ragwort (Senecio erraticus) in sheep. Vet. Human Toxicol. 25(1):4-7.

Barros C.L.S., Metzdorf L.L., Santos M.N., Barros S.S. \& Peixoto P.V. 1989. Intoxicação experimental por Senecio brasiliensis (Compositae) em ovinos. Pesq.Vet. Bras. 9(3/4):55-67.

Bull L.B., Culvenor C.C.J. \& Dick A.T. 1968. The pyrrolizidine alkaloids:their chemistry, pathogenicity and other biological properties. North-Holland Publisher, Amsterdam. 293p.

Cruz C.E.F., Karam F.C., Dalto A.C., Pavarini S.P., Bandarra P.M. \& Driemeier D. 2010. Fireweed (Senecio madagascariensis) poisoning in cattle. Pesq. Vet. Bras. 30(1):10-12.

Cullen J.M. 2007. Liver, biliary system, and exocrine pancreas, p.426-446. In: McGavin M.D. \& Zachary J.F. (Eds), Pathologic Basis of Veterinary Disease. $4^{\text {th }}$ ed. Mosby Elsevier, St Louis, Missouri.

Dollahite J.W. 1972. The use of sheep and goats to control Senecio poisoning in cattle. South Vet. 25(3):223-226.

Forsyth A.A. 1979. British Poisonous Plants $2^{\text {nd }}$ ed. Ministry Agriculture Fish and Food. London, p.98-102.

Grecco F.B., Estima-Silva P., Marcolongo-Pereira C., Soares M.P., Collares G. \& Schild A.L. 2011. Seneciose crônica em ovinos no sul do Rio Grande do Sul. Pesq. Vet. Bras. 31(4):326-330.

Huan J., Miranda C.I., Buhler D.R. \& Cheeke P.R. 1998. Species differences in the hepatic microsomal enzyme metabolism of the pyrrolizidine alkaloids. Toxicol. Letters 99:127-137.

Ilha M.R., Loretti A.P., Barros S.S. \& Barros C.L.S. 2001. Intoxicação espontânea por Senecio brasiliensis (Asteraceae) em ovinos no Rio Grande do Sul. Pesq. Vet. Bras. 21:123-138.

Karam F.S.C., Méndez M.C., Jarenkow J.A. \& Riet-Correa F. 2002. Fenologia de quatro espécies tóxicas de Senecio (Asteraceae) na região sul do Rio Grande do Sul. Pesq. Vet. Bras. 22(1):33-39.

Kellerman T.S., Coetzer J.A.W. \& Naudé T.W. 2005. Plant Poisonings and Mycotoxicoses of Livestock in Southern Africa. $2^{\text {nd }}$ ed. Oxford University Press, Cape Town, p.6-13.

Laningan G.W. 1970. Metabolism of pyrrolizidine alkaloids in the ovine rumen. Aust. J. Agricult. Res. 22:123-130.

Méndez M.C. \& Riet-Correa F. 1993. Intoxication by Senecio tweediei in cattle in southern Brazil. Vet. Human Toxicol. 35(1):55.

Nobre V.M.T., Dantas A.F.M., Riet-Correa F., Barbosa Filho J.M., Tabosa I.M. \& Vasconcelos J.J. 2005. Acute intoxication by Crotalaria retusa in sheep. Toxicon 45:347-352.

Riet-Correa F. \& Medeiros R.M.T. 2001. Intoxicação por plantas em ruminantes no Brasil e no Uruguai: importância econômica, controle e riscos para a saúde pública. Pesq. Vet. Bras. 21(1):38-42.

Rissi D.R., Rech R.R., Pierezan F., Gabriel A.L., Trost M.E., Brum J.S., Kommers G.D. \& Barros C.S.L. 2007. Intoxicações por plantas e micotoxinas associadas a plantas em bovinos no Rio Grande do Sul: 461 casos. Pesq. Vet. Bras. 27(7):261-268.

Seaman J.T. 1987. Pyrrolizidine alkaloid poisoning of sheep in New South Wales. Aust. Vet. J. 64:164-167. 